\title{
Psychiater in Facharzt für psychische Erkrankungen oder für psychosoziale Medizin umbenennen - Pro
}

\author{
Rename Psychiatrist as Specialist for Mental Diseases or as Specialist \\ for Psychosocial Medicine - Pro
}

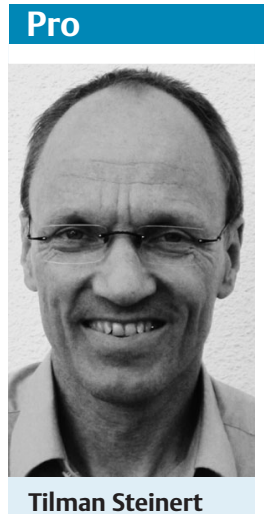

Tilman Steinert
Die Psychiatrie hat viele Fortschritte im Verständnis und in der Behandlung psychischer Störungen erreicht, auf die wir stolz sein dürfen, zumal in den letzten Jahrzehnten. Ein großer Gewinn war die Integration der Psychotherapie in die Praxis, in die Weiterbildungsordnung und damit letztlich auch in das Selbstbild des Psychiaters bzw. heute bereits überwiegend der Psychiaterin. Berufsbezeichnungen sind identitätsbildend und sollten nicht nach Tagesbefindlichkeiten geändert werden. Der Psychiater ist gemäß der aus dem Griechischen stammenden Wortbedeutung nichts anderes als ein „Arzt für psychische Erkrankungen“ und kann auf eine über 200-jährige, im Wesentlichen durch medizinischen Fortschritt gekennzeichnete Geschichte zurückblicken, seit Johann Christian Reil den Begriff der „Psychiaterie“ 1808 erstmals verwendete [1].

All dies ist richtig. Dem stehen auf der anderen Seite der Waagschale aber gewichtige Argumente entgegen, sich doch von der Psychiatrie sprachlich zunehmend zu verabschieden. Alle Imagekampagnen und Entstigmatisierungsprogramme konnten nicht verhindern, dass in der Öffentlichkeit Psychiatrie noch immer - vielleicht auch seit dem Fall Mollath noch mehr - mit forensischer Psychiatrie, Behandlung von Straftätern und Einsperren in Verbindung gebracht wird. Die öffentliche Wahrnehmung von jährlich über 1,1 Millionen stationären psychiatrischen Behandlungsfällen in Deutschland [2] wird maßgeblich durch eine Handvoll langzeituntergebrachter Sexualstraftäter im Maßregelvollzug, von Menschen in Sicherungsverwahrung und einem sehr geringen Anteil von Patienten bestimmt, die nach einer Begutachtung und einer Ge- richtsentscheidung eine Zwangsmedikation erhalten. $\mathrm{Zu}$ diesem Ballast durch eine Verzerrung der Wahrnehmung in der Gegenwart kommt der tatsächliche Ballast der Vergangenheit hinzu. Folterähnliche Zwangsmaßnahmen als „Therapie“ in der ersten Hälfte des 19. Jahrhunderts eng verknüpft mit der Entstehung des Fachgebiets, erneut während des Ersten Weltkriegs als Militärpsychiatrie, therapeutischer Nihilismus in der zweiten Hälfte des 19. Jahrhunderts, Hungersterben in den Anstalten im Ersten Weltkrieg, Zwangssterilisationen auf der Basis wissenschaftlich falscher eugenischer, von führenden Psychiatern vertretener Theorien, die Massenmorde an psychisch Kranken während der Zeit des Zweiten Weltkriegs ebenfalls unter Beteiligung führender Psychiater, die nobelpreisgekrönten grausamen Verstümmelungen durch die präfrontale Lobotomie, „elende und menschenunwürdige Umstände“ in der deutschen Nachkriegspsychiatrie, aber auch in vielen anderen Teilen der Welt, Missbrauch der Psychiatrie in der Sowjetunion als politisches Disziplinierungsinstrument - die Liste ist unvollständig, aber sie sollte genügen. Eine Umbenennung des Facharztes, nebenbei in eine deutsche und allgemein verständliche Bezeichnung gleichen Bedeutungsgehalts, wäre auch die Chance zu einer Distanzierung von einer historischen Tradition, die problematischer und belasteter ist als bei allen anderen medizinischen Fächern.

Beispiele für derartig intendierte Umbenennungen gibt es genug. Das Argument, dass es nur eine Frage der Zeit ist, bis dasselbe Stigma sich an den neuen Begriff gehaftet hat, ist vordergründig und in vielen Fällen auch gut widerlegt. Die Benennung diskriminierter gesellschaftlicher Minderheiten wurde, einem breiten Konsensus von Political Correctness folgend, in den letzten Jahren geändert. „Neger“ und „Zigeuner" sind aus dem Sprachgebrauch verschwunden. In welchem Ausmaß der Sprachgebrauch auch das Denken be- stimmt, ist Gegenstand einer umfangreichen Literatur, angefangen vom linguistischen Klassiker „Sprache - Denken Wirklichkeit" von Benjamin Whorf [3] und zum Beispiel bei Gender-Fragen oder dem Umgang mit sexuellen Minderheiten in allen Verästelungen nachzulesen und in den Änderungen unseres Alltagslebens anschaulich zu verfolgen.

Auch in der Psychiatrie ist eine prominente Umbenennung zumindest in der Diskussion. Der Begriff „Schizophrenie“ hätte vor Kurzem (2011) 100-jähriges Jubiläum feiern können. Gefeiert wurde nicht, anstelle dessen gibt es vielfältige Kritik in Bezug auf Stigma und mangelnde wissenschaftliche Fundierung. Als Ergebnis einer bereits in den 1990er-Jahren begonnenen Kampagne beschloss die japanische psychiatrische Gesellschaft Anfang des Jahrtausends nach umfänglichen Diskussionen, den Begriff Schizophrenie aufzugeben und durch den Begriff Togo-Shitsho Sho (Integrations-Dysregulations-Syndrom) zu ersetzen [4-6]. Ähnliche Initiativen gibt es z.B. in den Niederlanden, Neuseeland und Großbritannien vorwiegend von Verbänden der Betroffenen und Angehörigen, verschiedene Namen wurden vorgeschlagen $[7,8]$. In Japan konnte inzwischen gezeigt werden, dass von College-Studenten die neue Bezeichnung tatsächlich weniger mit kriminellem Verhalten assoziiert wurde als die alte [9]. Entstigmatisierung und Änderung der Sinnzuschreibung via Sprachgebrauch ist also weder reine Fiktion noch bloßer Euphemismus, sondern hinsichtlich der Auswirkungen empirisch belegbar.

Auch psychiatrische Institutionen können ohne Identitätsverlust und Geschichtsleugnung anders heißen. Eng verknüpft mit dem Beginn der Reformpsychiatrie in Deutschland ist bekanntlich die Gründung eines „Zentralinstituts für Seelische Gesundheit“" in Mannheim [10], das sich eines hervorragenden Rufs erfreut. Schließlich ändern sich auch Berufsbezeichnungen. Aus der Sprechstundenhilfe ist eine medizinische Fachangestellte ge- 
worden, aus dem Wundarzt und Feldscher schon länger ein Chirurg, aus dem Pädiater ein Arzt für Kinder- und Jugendmedizin. Die Zeit wäre reif für eine Umbenennung des Facharztes für Psychiatrie und Psychotherapie in einen Facharzt für psychische Erkrankungen - als Signal an die nachfolgenden Kollegen, die Patienten, die Öffentlichkeit und nicht zuletzt uns selbst. Zumal wir in Deutschland die „Psychiaterie“ begrifflich eingeführt haben, könnten wir auch an dieser Stelle 200 Jahre später wieder vorangehen. Es gibt nur ein wirkliches Hindernis. Ein Nebeneinander von einem „Arzt für psychische Erkrankungen“ und einem „Arzt für psychosomatische Medizin und Psychotherapie“ macht noch weniger Sinn als das derzeitige Nebeneinander. Insofern wäre die Herausforderung eine doppelte: die eigene Identität infrage zu stellen und zugleich einen tragfähigen Konsens (im Sinne eines „Common Trunk“) im schwierigen Verhältnis zu den in ihrer Identität weltweit auch einzigartigen und daher auf fragwürdigem Grund stehenden Fachgeschwistern für psychosomatische Medizin zu finden. Zumal eine einfache Annexion und Einverleibung dieses anderen Facharztes nicht zur Disposition steht, müsste freilich das Selbstverständnis wirklich grundlegend in die Diskus- sion eingebracht werden. Die Psychosomatiker haben das Problem mit dem historischen und begrifflichen Stigma für sich scheinbar bereits gelöst und sind deshalb reserviert. Dennoch - umso größer wäre der Gewinn, wenn es gelingen würde, am besten im Zuge der Verabschiedung einer ohnehin geforderten neuen Weiterbildungsordnung. Damit wäre auch gesichert, dass es am Ende um das noch Wichtigere geht: nicht die Verpackung, sondern was drin ist und wie wir das leben.

\section{Literatur}

1 Marneros A, Pillmann F. Das Wort Psychiatrie... wurde in Halle geboren. Von den Anfängen der deutschen Psychiatrie. Stuttgart, New York: Schattauer; 2005

2 Schneider F, Falkai P, Maier W. Psychiatrie 2020. Berlin, Heidelberg: Springer; 2011

3 Whorf BL. Sprache, Denken, Wirklichkeit. Beiträge zur Metalinguistik und Sprachphilosophie. Reinbek: Rowohlt; 1963

4 Sato M. Renaming schizophrenia. Japanese perspective. World Psychiatry 2006; 5: 53 55

5 van Os J. 'Salience syndrome' replaces ‘schizophrenia' in DSM-5 and ICD-11. Psychia-

Sie haben eine eigene Meinung zu diesem

Thema? Dann schreiben Sie uns an:

psychiat-praxis@thieme.de! try's evidence-based entry into the 21st century? Acta Psychiatr Scand 2009; 120: $363-372$

6 Steinert T. Keine Laufzeitverlängerung für Schizophrenie? Psychiat Prax 2010; 37: $46-48$

7 George B, Klijn A. A modern name for schizophrenia (PSS) would diminish self-stigma. Psychol Med 2013; 43: 1555 - 1567

8 Brabban A, Morrison T, Read J. Renaming 'schizophenia': a step too far or not far enough? Psychol Med 2013; 43: 1558 1560

9 Takahashi H, Ideno T, Okubo S et al. Impact of chancing the Japanese term for 'schizophrenia' for reasons of stereotypical beliefs of schizophrenia in Japanese youth. Schizophrenia Res 2009; 112: 149-152

10 Häfner $H$, Martini $H$. Das Zentralinstitut für seelische Gesundheit. Gründungsgeschichte und Gegenwart. München: C. H. Beck; 2011

Korrespondenzadresse

Prof. Dr. med. Tilman Steinert

Zentrum für Psychiatrie Südwürttemberg

Weingartshoferstraße 2

88214 Ravensburg-Weissenau

tilman.steinert@zfp-zentrum.de

Bibliografie

Dol http://dx.doi.org/

10.1055/s-0033-1349615

Psychiat Prax 2014; 41: 11-12

(c) Georg Thieme Verlag KG

Stuttgart · New York

ISSN 0303-4259 